\title{
New results from DAMA/LIBRA
}

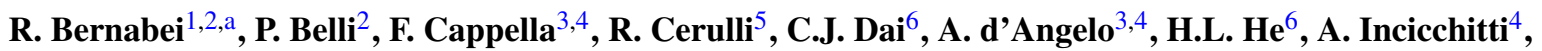 \\ H.H. Kuang ${ }^{6}$, X.H. Ma ${ }^{6}$, F. Montecchia ${ }^{2,7}$, F. Nozzoli ${ }^{1,2}$, D. Prosperi ${ }^{3,4}$, X.D. Sheng ${ }^{6}$, R.G. Wang ${ }^{6}$, Z.P. Ye ${ }^{6,8}$ \\ ${ }^{1}$ Dipartimento di Fisica, Università di Roma “Tor Vergata”, 00133 Rome, Italy \\ ${ }^{2}$ INFN, sez. Roma "Tor Vergata", 00133 Rome, Italy \\ ${ }^{3}$ Dipartimento di Fisica, Università di Roma "La Sapienza", 00185 Rome, Italy \\ ${ }^{4}$ INFN, sez. Roma, 00185 Rome, Italy \\ ${ }^{5}$ Laboratori Nazionali del Gran Sasso, I.N.F.N., Assergi, Italy \\ ${ }^{6}$ IHEP, Chinese Academy, P.O. Box 918/3, Beijing 100039, China \\ ${ }^{7}$ Lab. Sperim. Policentrico di Ingegneria Medica, Università di Roma "Tor Vergata", 00133 Rome, Italy \\ ${ }^{8}$ University of Jing Gangshan, Jiangxi, China
}

Received: 10 February 2010 / Revised: 12 March 2010 / Published online: 23 March 2010

(C) Springer-Verlag / Società Italiana di Fisica 2010

\begin{abstract}
DAMA/LIBRA is running at the Gran Sasso National Laboratory of the I.N.F.N. Here the results obtained with a further exposure of 0.34 ton $\times \mathrm{yr}$ are presented. They refer to two further annual cycles collected one before and one after the first DAMA/LIBRA upgrade occurred on September/October 2008. The cumulative exposure with those previously released by the former DAMA/NaI and by DAMA/LIBRA is now 1.17 ton $\times \mathrm{yr}$, corresponding to 13 annual cycles. The data further confirm the previous positive results obtained investigating the presence of Dark Matter (DM) particles in the galactic halo by means of the model independent Dark Matter annual modulation signature; the confidence level is now $8.9 \sigma$ for the cumulative exposure. In particular, with the cumulative exposure the modulation amplitude of the single-hit events in the (2-6) $\mathrm{keV}$ energy interval measured in $\mathrm{NaI}(\mathrm{Tl})$ target is $(0.0116 \pm 0.0013)$ $\mathrm{cpd} / \mathrm{kg} / \mathrm{keV}$; the measured phase is $(146 \pm 7)$ days and the measured period is $(0.999 \pm 0.002) \mathrm{yr}$, values well in agreement with those expected for the DM particles.
\end{abstract}

\section{Introduction}

The former DAMA/NaI [1-30] and the present DAMA/ LIBRA [31-33] experiments at the Gran Sasso National Laboratory have the main aim to investigate the presence of Dark Matter particles in the galactic halo by exploiting the model independent Dark Matter annual modulation signature originally suggested in the mid 80's in Refs. [34, 35]. In fact, as a consequence of its annual revolution around the

a e-mail: rita.bernabei@roma2.infn.it
Sun, which is moving in the Galaxy traveling with respect to the Local Standard of Rest towards the star Vega near the constellation of Hercules, the Earth should be crossed by a larger flux of Dark Matter particles around $\sim 2$ June (when the Earth orbital velocity is summed to the one of the solar system with respect to the Galaxy) and by a smaller one around $\sim 2$ December (when the two velocities are subtracted). Thus, this signature has a different origin and peculiarities than the seasons on the Earth and than effects correlated with seasons (consider the expected value of the phase as well as the other requirements listed below). This annual modulation signature is very distinctive since the effect induced by DM particles must simultaneously satisfy all the following requirements: the rate must contain a component modulated according to a cosine function (1) with one year period (2) and a phase that peaks roughly around $\simeq 2$ nd June (3); this modulation must only be found in a well-defined low energy range, where DM particle induced events can be present (4); it must apply only to those events in which just one detector of many actually "fires" (singlehit events), since the DM particle multi-interaction probability is negligible (5); the modulation amplitude in the region of maximal sensitivity must be $\lesssim 7 \%$ for usually adopted halo distributions (6), but it can be larger in case of some possible scenarios such as e.g. those in Refs. [36-40]. This offers an efficient DM model independent signature, able to test a large interval of cross sections and of halo densities; moreover, the use of highly radiopure $\mathrm{NaI}(\mathrm{Tl})$ scintillators as target-detectors assures sensitivity to wide ranges of DM candidates, of interaction types and of astrophysical scenarios.

It is worth noting that only systematic effects or side reactions able to simultaneously fulfill all the 6 requirements 
given above (and no one has ever been suggested) and to account for the whole observed modulation amplitude might mimic this DM signature.

The DAMA/LIBRA set-up, whose description, radiopurity and main features are discussed in details in Ref. [32] has firstly been upgraded in September/October 2008: (i) one detector has been recovered by replacing a broken PMT (see Ref. [31]); (ii) a new optimization of some PMTs and HVs has been performed; (iii) all the transient digitizers recording the shape of the pulse have been replaced with new ones, the U1063A Acqiris 8-bit 1GS/s DC270 HighSpeed cPCI Digitizers; (iv) a new DAQ with optical read-out has been installed. Also during this upgrade the operations involving the handling of the sensitive part of the setup and the shield have been performed in HP Nitrogen atmosphere. The upgrade has allowed to enlarge the sensitive mass and to improve general features. Here we just remind that the sensitive part of this set-up is made of 25 highly radiopure $\mathrm{NaI}(\mathrm{Tl})$ crystal scintillators (5-rows by 5 -columns matrix) having $9.70 \mathrm{~kg}$ mass each one. In each detector two $10 \mathrm{~cm}$ long special quartz light guides act also as optical windows on the two end faces of the crystal and are coupled to two low background photomultipliers working in coincidence at single photoelectron level. The detectors are housed in a sealed low-radioactive copper box installed in the center of a low-radioactive $\mathrm{Cu} / \mathrm{Pb} / \mathrm{Cd}$-foils/polyethylene/paraffin shield; moreover, about $1 \mathrm{~m}$ concrete (made from the Gran Sasso rock material) almost fully surrounds (mostly outside the barrack) this passive shield, acting as a further neutron moderator. A threefold-levels sealing system excludes the detectors from the environmental air of the underground laboratory [32]. A hardware/software system to monitor the running conditions is operative and self-controlled computer processes automatically control several parameters and manage alarms. Moreover: (i) the light response ranges from 5.5 to 7.5 photoelectrons $/ \mathrm{keV}$, depending on the detector; (ii) the hardware threshold of each PMT is at single photoelectron (each detector is equipped with two low back-

Table 1 Exposures of the DAMA/LIBRA-5,6 annual cycles. Here $\alpha=\left\langle\cos ^{2} \omega\left(t-t_{0}\right)\right\rangle$ is the mean value of the squared cosine and $\beta=\left\langle\cos \omega\left(t-t_{0}\right)\right\rangle$ is the mean value of the cosine (the averages are taken over the live time of the data taking and $t_{0}=152.5$ day, i.e. June ground photomultipliers working in coincidence); (iii) energy calibration with $\mathrm{X}$-rays $/ \gamma$ sources are regularly carried out down to few $\mathrm{keV}$; (iv) the software energy threshold of the experiment is $2 \mathrm{keV}$ electron equivalent (hereafter $\mathrm{keV}$ ); (v) both single-hit events (where just one of the detectors fires) and multiple-hit events (where more than one detector fires) are acquired; (v) the data are collected up to the $\mathrm{MeV}$ region despite the optimization is performed for the lower one. For the radiopurity, the procedures and further details see Refs. [31, 32].

The data of the former DAMA/NaI (0.29 ton $\times \mathrm{yr})$ and those of the first 4 annual cycles of DAMA/LIBRA (total exposure 0.53 ton $\times$ yr) have already given positive model independent evidence for the presence of DM particles in the galactic halo with high confidence level on the basis of the DM annual modulation signature [31].

In this paper the model independent results with other two annual cycles DAMA/LIBRA-5,6 are presented. As mentioned, the data of the first cycle have been collected in the same conditions as DAMA/LIBRA-1,2,3,4 [31, 32], while the data of DAMA/LIBRA- 6 have been taken after the above mentioned 2008 upgrade.

\section{The results}

The updated exposures of the DAMA/LIBRA annual cycles and the cumulative one with the former DAMA/NaI are given in Table 1.

The only data treatment, which is performed on the raw data, is to remove noise pulses (mainly PMT noise, Cherenkov light in the light guides and in the PMT windows, and afterglows) near the energy threshold in the single-hit events; for a description of the used procedure and details see Ref. [32].

In the DAMA/LIBRA-1,2,3,4,5,6 annual cycles about $7.2 \times 10^{7}$ events have also been collected for energy calibrations and about $3 \times 10^{6}$ events $/ \mathrm{keV}$ for the evaluation of

2nd); thus, $\left(\alpha-\beta^{2}\right)$ indicates the variance of the cosine (i.e. it is 0.5 for a full year of data taking). The information on the previously published DAMA/LIBRA-1,2,3,4, are recalled as well as the cumulative exposure, when including the former DAMA/NaI

\begin{tabular}{lllll}
\hline & Period & $\begin{array}{l}\text { Mass } \\
(\mathrm{kg})\end{array}$ & $\begin{array}{l}\text { Exposure } \\
(\mathrm{kg} \times \text { day })\end{array}$ \\
\hline DAMA/LIBRA-1,2,3,4 & Sept. 9, 2003-July 17, 2007 & 232.8 & 192,824 & 0.537 \\
DAMA/LIBRA-5 & July 17, 2007-Aug. 29, 2008 & 232.8 & 66,105 & 0.468 \\
DAMA/LIBRA-6 & Nov. 12, 2008-Sept. 1, 2009 & 242.5 & 58,768 & 0.519 \\
DAMA/LIBRA-1 to -6 & Sept. 9, 2003-Sept. 1, 2009 & & $317697 \simeq 0.87$ ton $\times$ yr \\
DAMA/NaI + DAMA/LIBRA-1 to 6 & & 1.17 ton $\times$ yr \\
\hline
\end{tabular}


2-4 keV

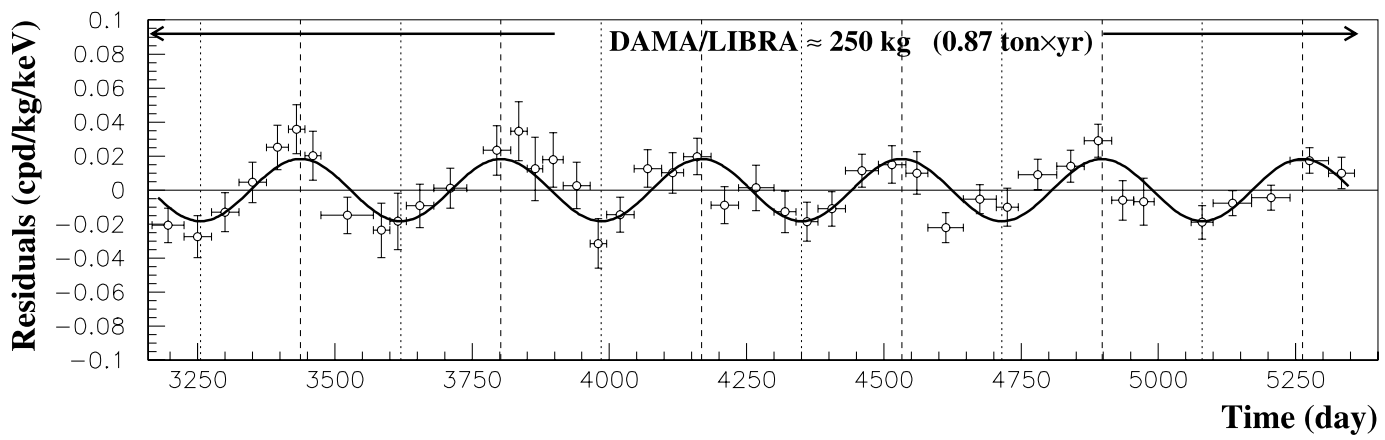

2-5 keV

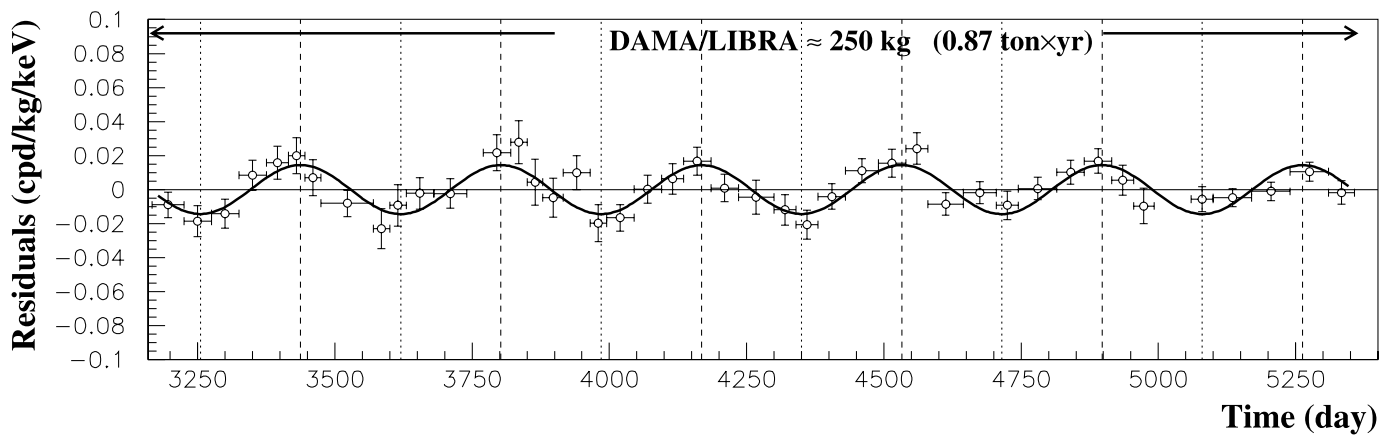

2-6 keV

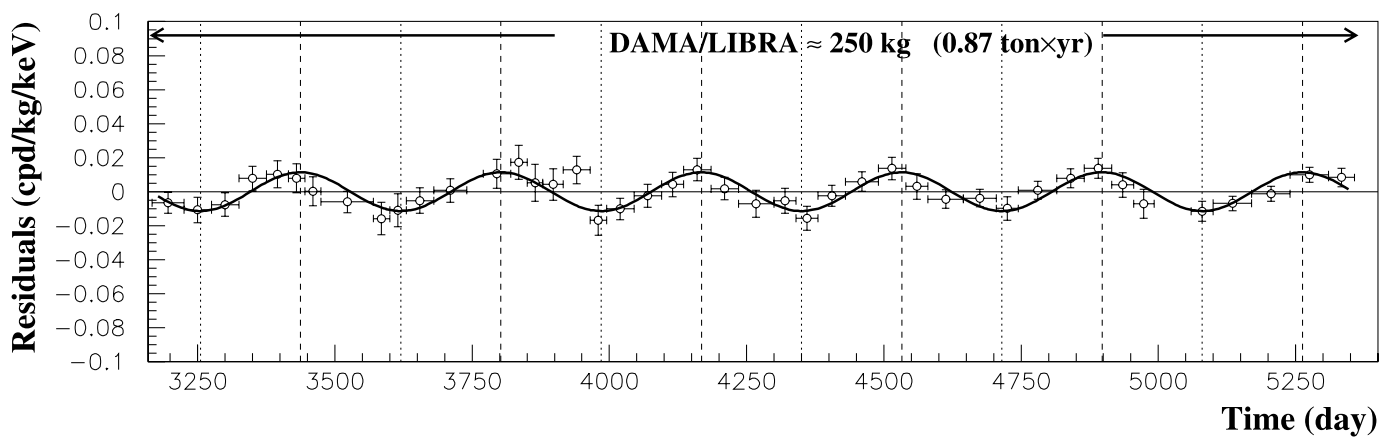

Fig. 1 Experimental model-independent residual rate of the single-hit scintillation events, measured by DAMA/LIBRA, 1,2,3,4,5,6 in the (2$4),(2-5)$ and (2-6) keV energy intervals as a function of the time. The zero of the time scale is January 1st of the first year of data taking of the former DAMA/NaI experiment [31]. The experimental points present the errors as vertical bars and the associated time bin width as horizontal bars. The superimposed curves are the cosinusoidal functions behaviors $A \cos \omega\left(t-t_{0}\right)$ with a period $T=\frac{2 \pi}{\omega}=1 \mathrm{yr}$, with a phase $t_{0}=152.5$ day (June 2nd) and with modulation amplitudes, $A$, equal to the central values obtained by best fit over the whole data including also the exposure previously collected by the former DAMA/NaI experiment: cumulative exposure is 1.17 ton $\times \mathrm{yr}$ (see also Ref. [31] and references therein). The dashed vertical lines correspond to the maximum expected for the DM signal (June 2nd), while the dotted vertical lines correspond to the minimum. See text

performed as previously done in Ref. [31] and references therein. In particular, Fig. 1 shows the time behavior of the experimental residual rates for single-hit events in the (2$4),(2-5)$ and (2-6) keV energy intervals. These residual rates are calculated from the measured rate of the singlehit events (already corrected for the overall efficiency and for the acquisition dead time) after subtracting the constant part: $\left\langle r_{i j k}-f l a t_{j k}\right\rangle_{j k}$. Here $r_{i j k}$ is the rate in the considered $i$-th time interval for the $j$-th detector in the $k$-th energy bin, while $f l a t_{j k}$ is the rate of the $j$-th detector in the $k$-th
Several analyses on the model-independent investigation of the DM annual modulation signature have been 
Table 2 Test of absence of modulation in the data of the DAMA/LIBRA-1,2,3,4,5,6 and without/with also the data of the former DAMA/NaI. As it can be seen, a null modulation amplitude is discarded by the data

\begin{tabular}{lll}
\hline $\begin{array}{l}\text { Energy interval } \\
(\mathrm{keV})\end{array}$ & $\begin{array}{l}\text { DAMA/LIBRA } \\
(6 \text { annual cycles })\end{array}$ & $\begin{array}{l}\text { DAMA/NaI \& DAMA/LIBRA } \\
(7+6 \text { annual cycles })\end{array}$ \\
\hline $2-4$ & $\chi^{2} /$ d.o.f. $=90.0 / 43 \rightarrow \mathrm{P}=3.6 \times 10^{-5}$ & $\chi^{2} /$ d.o.f. $=147.4 / 80 \rightarrow \mathrm{P}=6.8 \times 10^{-6}$ \\
$2-5$ & $\chi^{2} /$ d.o.f. $=82.1 / 43 \rightarrow \mathrm{P}=3.1 \times 10^{-4}$ & $\chi^{2} /$ d.o.f. $=135.2 / 80 \rightarrow \mathrm{P}=1.1 \times 10^{-4}$ \\
$\chi^{2} /$ d.o.f. $=68.9 / 43 \rightarrow \mathrm{P}=7.4 \times 10^{-3}$ & $\chi^{2} /$ d.o.f. $=139.5 / 80 \rightarrow \mathrm{P}=4.3 \times 10^{-5}$ \\
\hline
\end{tabular}

Table 3 Modulation amplitude, A, obtained by fitting the single-hit residual rate of the six DAMA/LIBRA annual cycles (Fig. 1), and including also the former DAMA/NaI data given elsewhere (see [31] and references therein) for a total cumulative exposure of 1.17 ton $\times$ yr. It has been obtained by fitting the data with the formula: $A \cos \omega\left(t-t_{0}\right)$ with $T=\frac{2 \pi}{\omega}=1 \mathrm{yr}$ and $t_{0}=152.5$ day (June 2 nd), as expected for a signal by the DM annual modulation signature. The corresponding $\chi^{2}$ value for each fit and the confidence level are also reported

\begin{tabular}{lll}
\hline $\begin{array}{l}\text { Energy interval } \\
(\mathrm{keV})\end{array}$ & $\begin{array}{l}\text { DAMA/LIBRA } \\
(\mathrm{cpd} / \mathrm{kg} / \mathrm{keV})\end{array}$ & $\begin{array}{l}\text { DAMA/NaI \& DAMA/LIBRA } \\
(\mathrm{cpd} / \mathrm{kg} / \mathrm{keV})\end{array}$ \\
\hline $2-4$ & $\mathrm{~A}=(0.0170 \pm 0.0024)$ & $\mathrm{A}=(0.0183 \pm 0.0022)$ \\
& $\chi^{2} /$ d.o.f. $=75.7 / 79 \rightarrow 8.3 \sigma$ C.L. $=41.0 / 42$ & $\mathrm{~A}=(0.0144 \pm 0.0016)$ \\
$2-5$ & $\mathrm{~A}=(0.0129 \pm 0.0018)$ & $\chi^{2} /$ d.o.f. $=56.6 / 79 \rightarrow 9.0 \sigma$ C.L. \\
$2-6$ & $\chi^{2} /$ d.o.f. $=30.7 / 42$ & $\mathrm{~A}=(0.0114 \pm 0.0013)$ \\
& $\mathrm{A}=(0.0097 \pm 0.0015)$ & $\chi^{2} /$ d.o.f. $=64.7 / 79 \rightarrow 8.8 \sigma$ C.L. \\
\hline
\end{tabular}

energy bin averaged over the cycles. The average is made on all the detectors ( $j$ index) and on all the energy bins $(k$ index) which constitute the considered energy interval. The weighted mean of the residuals must obviously be zero over one cycle.

For clarity in Fig. 1 only the DAMA/LIBRA data collected over six annual cycles (0.87 ton $\times$ yr) are shown; the DAMA/NaI data ( 0.29 ton $\times$ yr) and comparison with DAMA/LIBRA are available in Ref. [31].

The hypothesis of absence of modulation in the data can be discarded (see Table 2).

The single-hit residual rate of DAMA/LIBRA-1,2,3,4,5,6 of Fig. 1 can be fitted with the formula: $A \cos \omega\left(t-t_{0}\right)$ considering a period $T=\frac{2 \pi}{\omega}=1 \mathrm{yr}$ and a phase $t_{0}=152.5$ day (June 2nd), as expected by the DM annual modulation signature; this can be repeated for the total available exposure 1.17 ton $\times$ yr including the former DAMA/NaI data (see [31] and references therein). The results are shown in Table 3 .

The compatibility among the 13 annual cycles has been investigated. In particular, the modulation amplitudes measured in each annual cycle of the whole 1.17 ton $\times$ yr exposure have been analyzed as in Ref. [31]. Indeed these modulation amplitudes are normally distributed around their best fit value as pointed out by the $\chi^{2}$ test $\left(\chi^{2}=9.3,12.2\right.$ and 10.1 over 12 d.o.f. for the three energy intervals, respectively) and the run test (lower tail probabilities of $57 \%, 47 \%$ and $35 \%$ for the three en- ergy intervals, respectively). Moreover, the DAMA/LIBRA5 and DAMA/LIBRA-6 (2-6) keV modulation amplitudes are $(0.0086 \pm 0.0032) \mathrm{cpd} / \mathrm{kg} / \mathrm{keV}$ and $(0.0101 \pm$ $0.0031) \mathrm{cpd} / \mathrm{kg} / \mathrm{keV}$, respectively, in agreement with that of DAMA/LIBRA-1,2,3,4: (0.0110 \pm 0.0019$) \mathrm{cpd} / \mathrm{kg} / \mathrm{keV}$; we also recall that the statistical compatibility between the DAMA/NaI and DAMA/LIBRA-1,2,3,4 modulation amplitudes has been verified [31]. Thus, also when adding DAMA/LIBRA-5,6, the cumulative result from DAMA/NaI and DAMA/LIBRA can be adopted.

Table 4 shows the results obtained for the cumulative 1.17 ton $\times$ yr exposure when the period and phase parameters are kept free in the fitting procedure described above. The period and the phase are well compatible with expectations for a signal in the DM annual modulation signature. In particular, the phase-whose better determination will be achieved in the following by using a maximum likelihood analysis - is consistent with about June 2nd within $2 \sigma$; moreover, for completeness, we also note that a slight energy dependence of the phase could be expected in case of possible contributions of non-thermalized DM components to the galactic halo, such as e.g. the SagDEG stream [15] and the caustics [44].

The DAMA/LIBRA single-hit residuals of Fig. 1 and those of DAMA/NaI (see e.g. [31]) have also been investigated by a Fourier analysis, obtaining a clear peak corresponding to a period of 1 year (see Fig. 2); the same analysis in other energy region shows instead only aliasing peaks. 
Table 4 Modulation amplitude $(A)$, period $\left(T=\frac{2 \pi}{\omega}\right)$ and phase $\left(t_{0}\right)$, obtained by fitting, with the formula: $A \cos \omega\left(t-t_{0}\right)$, the single-hit residual rate of the cumulative 1.17 ton $\times$ yr exposure. The results are well compatible with expectations for a signal in the DM annual modulation signature

\begin{tabular}{|c|c|c|c|c|}
\hline Energy interval & $A(\mathrm{cpd} / \mathrm{kg} / \mathrm{keV})$ & $T=\frac{2 \pi}{\omega}(\mathrm{yr})$ & $t_{0}$ (days) & C.L. \\
\hline $2-4$ & $(0.0194 \pm 0.0022)$ & $(0.996 \pm 0.002)$ & $136 \pm 7$ & $8.8 \sigma$ \\
\hline $2-5$ & $(0.0149 \pm 0.0016)$ & $(0.997 \pm 0.002)$ & $142 \pm 7$ & $9.3 \sigma$ \\
\hline $2-6$ & $(0.0116 \pm 0.0013)$ & $(0.999 \pm 0.002)$ & $146 \pm 7$ & $8.9 \sigma$ \\
\hline
\end{tabular}

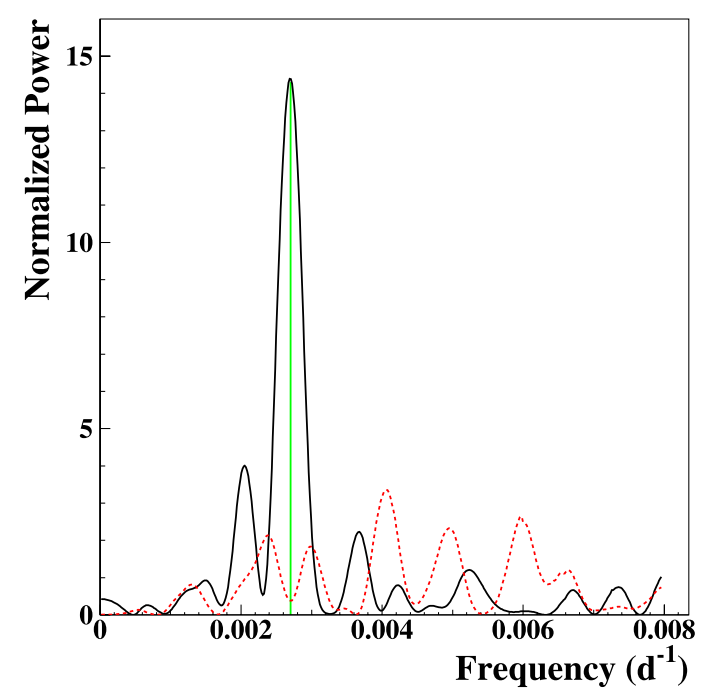

Fig. 2 Power spectrum of the measured single-hit residuals in the (2-6) keV (solid lines) and (6-14) keV (dotted lines) energy intervals calculated according to Refs. [41, 42], including also the treatment of the experimental errors and of the time binning. The data refer to: (a) DAMA/LIBRA-1,2,3,4,5,6 (exposure of 0.87 ton $\times$ yr); (b) the cumulative 1.17 ton $\times$ yr exposure (DAMA/NaI and DAMA/LIBRA-

The measured energy distribution has been investigated in other energy regions not of interest for Dark Matter, also verifying the absence of any significant background modulation. ${ }^{1}$ Following the procedures described in Ref. [31] and references therein, the measured rate integrated above $90 \mathrm{keV}, \mathrm{R}_{90}$, as a function of the time has been analyzed. In particular, also for these two latter annual cycles the distribution of the percentage variations of $\mathrm{R}_{90}$ with respect to the mean values for all the detectors has been considered; it shows a cumulative Gaussian behavior with $\sigma \simeq 1 \%$, well accounted by the statistical spread expected from the used sampling time (see Fig. 3). Moreover, fitting the time behavior of $\mathrm{R}_{90}$ with phase and period as for DM particles, a modulation amplitude compatible with zero is also found

\footnotetext{
${ }^{1}$ In fact, the background in the lowest energy region is essentially due to "Compton" electrons, X-rays and/or Auger electrons, muon induced events, etc., which are strictly correlated with the events in the higher energy part of the spectrum. Thus, if a modulation detected in the lowest energy region would be due to a modulation of the background (rather than to a signal), an equal or larger modulation in the higher energy regions should be present.
}

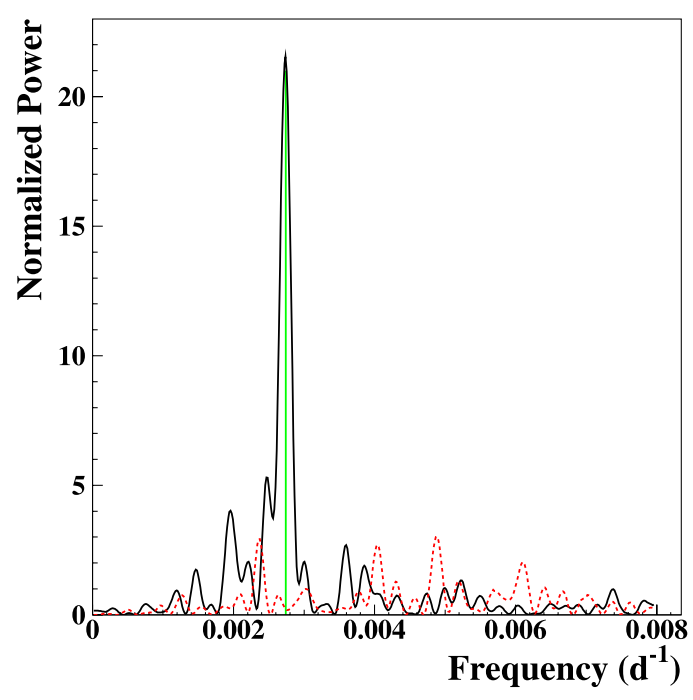

$1,2,3,4,5,6)$. As it can be seen, the principal mode present in the (2-6) $\mathrm{keV}$ energy interval corresponds to a frequency of $2.697 \times 10^{-3} \mathrm{~d}^{-1}$ and $2.735 \times 10^{-3} \mathrm{~d}^{-1}$ (vertical lines), respectively in the (a) and (b) case. They correspond to a period of $\simeq 1$ year. A similar peak is not present in the (6-14) keV energy interval just above

in DAMA/LIBRA-5 and DAMA/LIBRA-6: $(0.20 \pm 0.18)$ $\mathrm{cpd} / \mathrm{kg}$ and $(-0.20 \pm 0.16) \mathrm{cpd} / \mathrm{kg}$, respectively. This also excludes the presence of any background modulation in the whole energy spectrum at a level much lower than the effect found in the lowest energy region for the single-hit events. In fact, otherwise-considering the $\mathrm{R}_{90}$ mean values - a modulation amplitude of order of tens $\mathrm{cpd} / \mathrm{kg}$, that is $\simeq 100 \sigma$ far away from the measured value, would be present. Similar result is obtained when comparing the single-hit residuals in the (2-6) keV with those in other energy intervals; see as an example Fig. 4.

A further relevant investigation has been performed by applying the same hardware and software procedures, used to acquire and to analyze the single-hit residual rate, to the multiple-hit one. In fact, since the probability that a DM particle interacts in more than one detector is negligible, a DM signal can be present just in the single-hit residual rate. Thus, the comparison of the results of the single-hit events with those of the multiple-hit ones corresponds practically to compare between them the cases of DM particles beamon and beam-off. This procedure also allows an additional 
test of the background behavior in the same energy interval where the positive effect is observed. In particular, in Fig. 5 the residual rates of the single-hit events measured over the six DAMA/LIBRA annual cycles are reported, as collected in a single cycle, together with the residual rates of the multiple-hit events, in the considered energy intervals. While, as already observed, a clear modulation, satisfying all the peculiarities of the DM annual modulation signature, is present in the single-hit events, the fitted modulation amplitudes for the multiple-hit residual rate are well compatible with zero: $(-0.0011 \pm 0.0007) \mathrm{cpd} / \mathrm{kg} / \mathrm{keV}$, $(-0.0008 \pm 0.0005) \mathrm{cpd} / \mathrm{kg} / \mathrm{keV}$, and $(-0.0006 \pm 0.0004)$ $\mathrm{cpd} / \mathrm{kg} / \mathrm{keV}$ in the energy regions (2-4), (2-5) and (2-6)

Fig. 3 Distribution of the percentage variations of $\mathrm{R}_{90}$ with respect to the mean values for all the detectors in the DAMA/LIBRA-5,6 annual cycles (histogram); the superimposed curve is a Gaussian fit
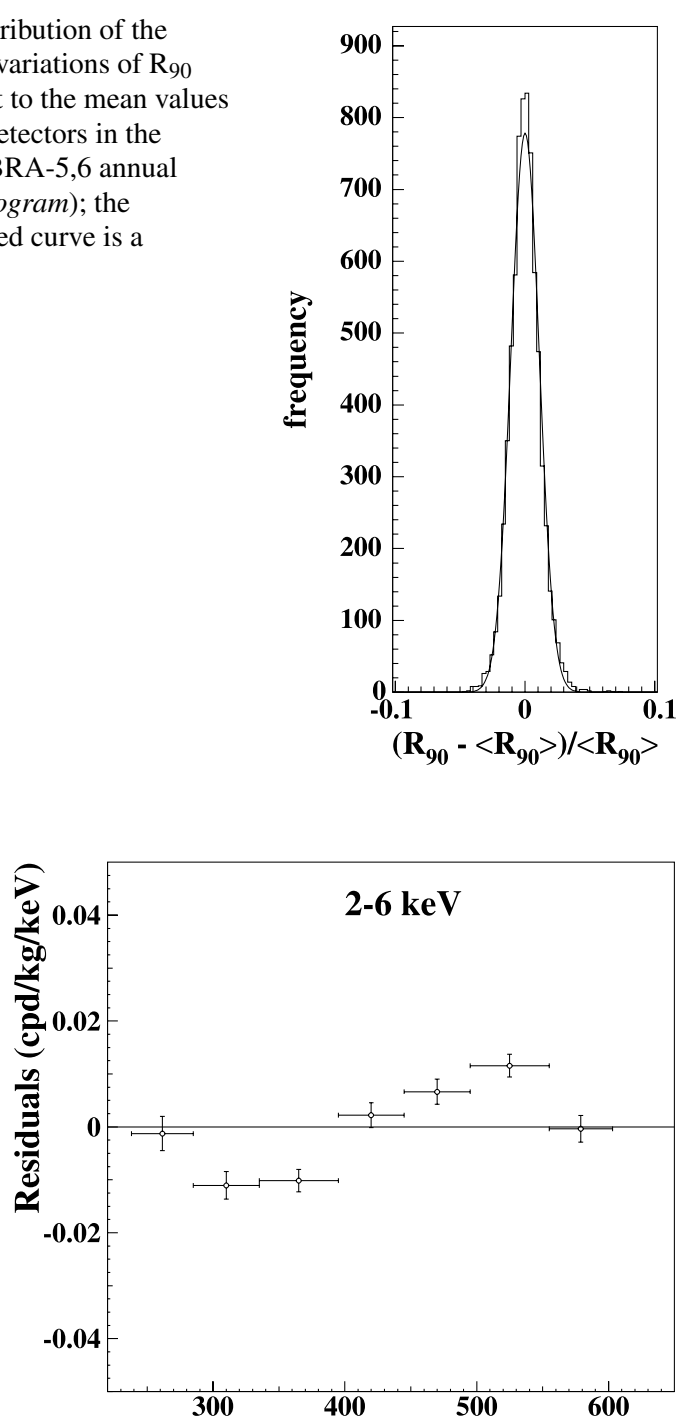

a)

Time (day)

Fig. 4 Experimental residuals in the (2-6) $\mathrm{keV}$ region and those in the (6-14) keV energy region just above for the cumulative 1.17 ton $\times$ yr, considered as collected in a single annual cycle. The experimental points present the errors as vertical bars and the associated time bin width as horizontal bars. The initial time of the figure is taken
$\mathrm{keV}$, respectively. Thus, again evidence of annual modulation with proper features as required by the DM annual modulation signature is present in the single-hit residuals (events class to which the DM particle induced events belong), while it is absent in the multiple-hit residual rate (event class to which only background events belong). Similar results were also obtained for the last two annual cycles of the DAMA/NaI experiment [13]. Since the same identical hardware and the same identical software procedures have been used to analyze the two classes of events, the obtained result offers an additional strong support for the presence of a DM particle component in the galactic halo. It is worth noting that the obtained results already account for whatever kind of background and, in addition, that no background process able to mimic the DM annual modulation signature (that is able to simultaneously satisfy all the peculiarities of the signature and to account for the measured modulation amplitude) is available (see also discussions e.g. in $[31,43])$.

As in Ref. [31], the annual modulation present at low energy can also be shown by depicting-as a function of the energy - the modulation amplitude, $S_{m, k}$, obtained by maximum likelihood method over the data considering $T=1 \mathrm{yr}$ and $t_{0}=152.5$ day. For such purpose the likelihood function of the single-hit experimental data in the $k$-th energy bin is defined as: $\mathbf{L}_{\mathbf{k}}=\Pi_{i j} e^{-\mu_{i j k}} \frac{\mu_{i j k}}{N_{i j k} !}$, where $N_{i j k}$ is the number of events collected in the $i$-th time interval (hereafter 1 day), by the $j$-th detector and in the $k$-th energy bin. $N_{i j k}$ follows a Poisson's distribution with expectation value $\mu_{i j k}=$ $\left[b_{j k}+S_{i k}\right] M_{j} \Delta t_{i} \Delta E \epsilon_{j k}$. The $b_{j k}$ are the background contributions, $M_{j}$ is the mass of the $j$-th detector, $\Delta t_{i}$ is the de-

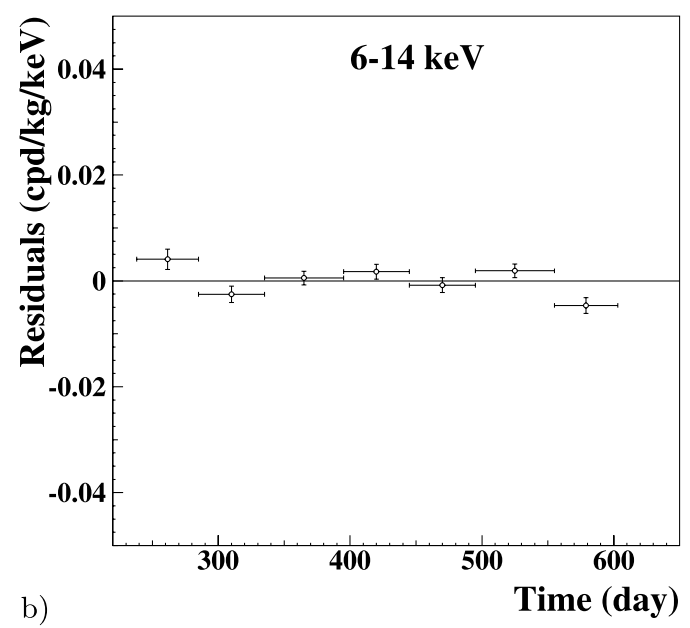

at August 7th. The clear modulation satisfying all the peculiarities of the DM annual modulation signature is present in the lowest energy interval, while it is absent just above; in fact, in the latter case the best fitted modulation amplitude is: $(0.00007 \pm 0.00077) \mathrm{cpd} / \mathrm{kg} / \mathrm{keV}$ 

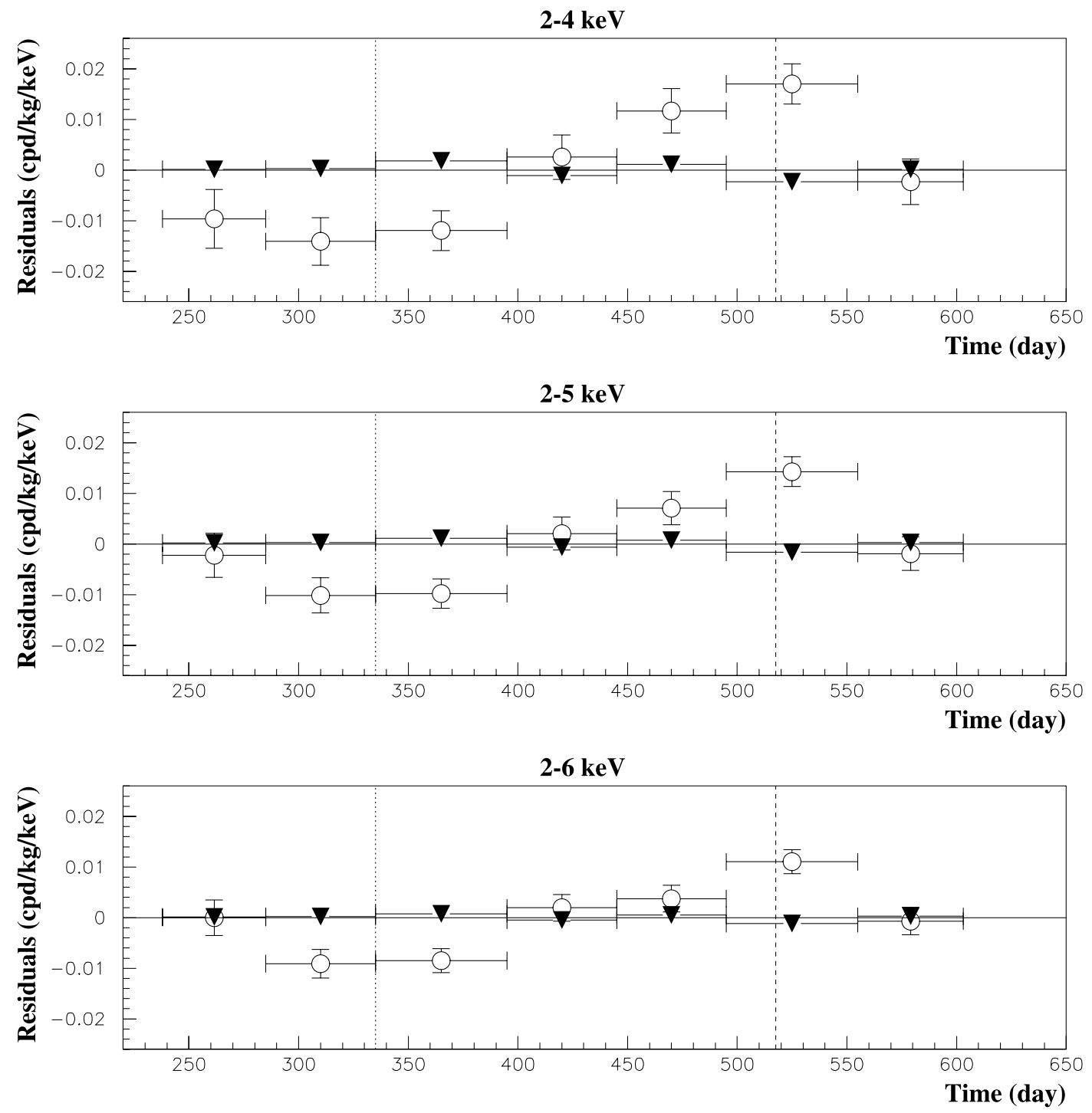

Fig. 5 Experimental residual rates over the six DAMA/LIBRA annual cycles for single-hit events (open circles) (class of events to which DM events belong) and for multiple-hit events (filled triangles) (class of events to which DM events do not belong). They have been obtained by considering for each class of events the data as collected in a single annual cycle and by using in both cases the same identical hardware

tector running time during the $i$-th time interval, $\Delta E$ is the chosen energy bin, $\epsilon_{j k}$ is the overall efficiency. Moreover, the signal can be written as $S_{i k}=S_{0, k}+S_{m, k} \cdot \cos \omega\left(t_{i}-t_{0}\right)$, where $S_{0, k}$ is the constant part of the signal and $S_{m, k}$ is the modulation amplitude. The usual procedure is to minimize the function $y_{k}=-2 \ln \left(\mathbf{L}_{\mathbf{k}}\right)-$ const for each energy bin; the free parameters of the fit are the $\left(b_{j k}+S_{0, k}\right)$ contributions and the $S_{m, k}$ parameter. Hereafter, the index $k$ is omitted when unnecessary.

In Fig. 6 the obtained $S_{m}$ are shown in each considered energy bin (there $\Delta E=0.5 \mathrm{keV}$ ). It can be inferred that positive signal is present in the (2-6) keV energy interval, and the same identical software procedures. The initial time of the figure is taken on August 7th. The experimental points present the errors as vertical bars and the associated time bin width as horizontal bars. See text and Ref. [31]. Analogous results were obtained for the DAMA/NaI data [13]

while $S_{m}$ values compatible with zero are present just above. In fact, the $S_{m}$ values in the (6-20) keV energy interval have random fluctuations around zero with $\chi^{2}$ equal to 27.5 for 28 degrees of freedom. All this confirms the previous analyses.

The method also allows the extraction of the $S_{m}$ values for each detector, for each annual cycle and for each energy bin. Thus, following the procedure described in Ref. [31], we have also verified that the $S_{m}$ are statistically well distributed in all the six DAMA/LIBRA annual cycles and in all the sixteen energy bins $(\Delta E=0.25 \mathrm{keV}$ in the $2-6 \mathrm{keV}$ energy interval) for each detector. Moreover, that procedure also allows the definition of a $\chi^{2}$ for each detector; the as- 
sociated degree of freedom are 16 for the detector restored after the upgrade in 2008 and 96 for the others. The values of the $\chi^{2} /$ d.o.f. range between 0.7 and 1.22 for twenty-four detectors, and the observed annual modulation effect is well distributed in all these detectors at 95\% C.L. A particular mention is deserved to the remaining detector whose value is 1.28 exceeding the value corresponding to that C.L.; this also is statistically consistent, considering that the expected number of detector exceeding this value over twenty-five is 1.25. Moreover, the mean value of the $25 \chi^{2} /$ d.o.f. is 1.066 , slightly larger than expected. Although this can be still ascribed to statistical fluctuations (see before), let us ascribe it to a possible systematics. In this case, one would have an additional error of $\leq 4 \times 10^{-4} \mathrm{cpd} / \mathrm{kg} / \mathrm{keV}$, if quadratically combined, or $\leq 5 \times 10^{-5} \mathrm{cpd} / \mathrm{kg} / \mathrm{keV}$, if linearly combined, to the modulation amplitude measured in the (2-6) $\mathrm{keV}$ energy interval. This possible additional error: $\leq 4 \%$

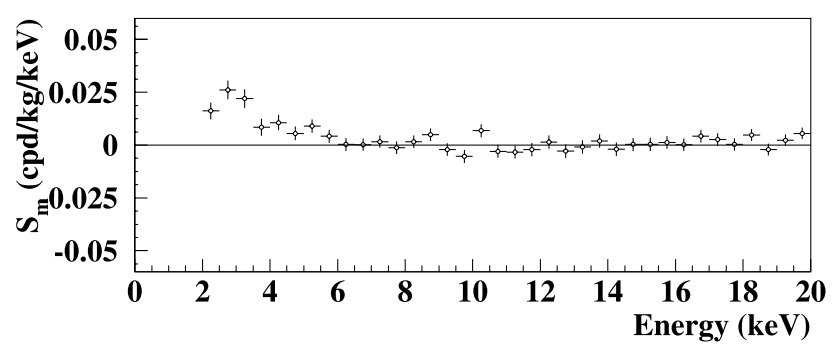

Fig. 6 Energy distribution of the $S_{m}$ variable for the total cumulative exposure 1.17 ton $\times$ yr. The energy bin is $0.5 \mathrm{keV}$. A clear modulation is present in the lowest energy region, while $S_{m}$ values compatible with zero are present just above. In fact, the $S_{m}$ values in the (6-20) $\mathrm{keV}$ energy interval have random fluctuations around zero with $\chi^{2}$ equal to 27.5 for 28 degrees of freedom

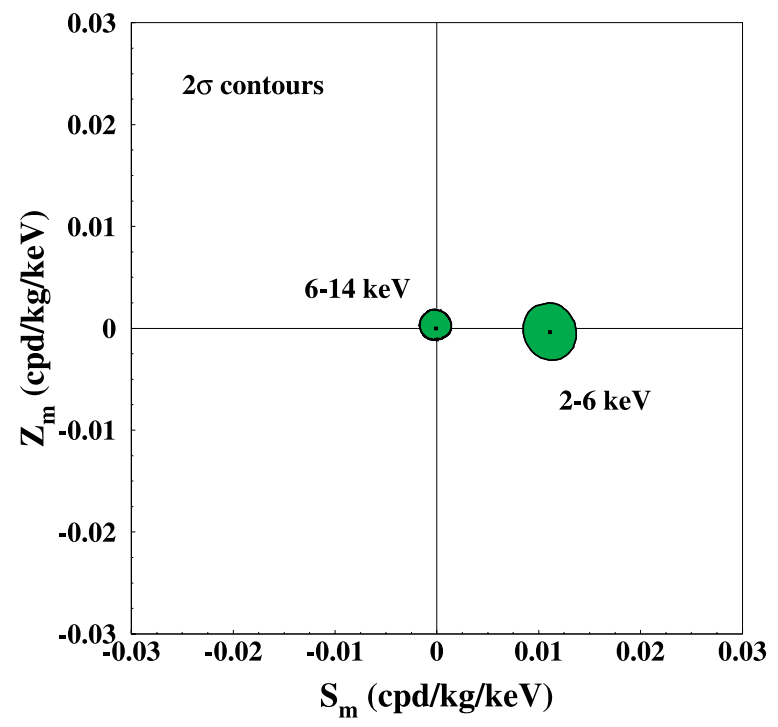

Fig. $72 \sigma$ contours in the plane $\left(S_{m}, Z_{m}\right)$ (left) and in the plane $\left(Y_{m}, t^{*}\right)$ (right) for the (2-6) $\mathrm{keV}$ and $(6-14) \mathrm{keV}$ energy intervals. The contours have been obtained by the maximum likelihood method, or $\leq 0.5 \%$, respectively, of the DAMA/LIBRA modulation amplitude is an upper limit of possible systematic effects.

Among further additional tests, the analysis of the modulation amplitudes as a function of the energy separately for the nine inner detectors and the remaining external ones has been carried out including the DAMA/LIBRA-5,6 data to those already analyzed in Ref. [31]. The obtained values are fully in agreement; in fact, the hypothesis that the two sets of modulation amplitudes as a function of the energy belong to same distribution has been verified by $\chi^{2}$ test, obtaining: $\chi^{2} /$ d.o.f. $=3.1 / 4$ and $7.1 / 8$ for the energy intervals (2-4) and (2-6) $\mathrm{keV}$, respectively $(\Delta E=0.5 \mathrm{keV})$. This shows that the effect is also well shared between inner and external detectors.

Let us, finally, release the assumption of a phase $t_{0}=$ 152.5 day in the procedure to evaluate the modulation amplitudes from the data of the 1.17 ton $\times$ yr. In this case alternatively the signal has been written as:

$$
\begin{aligned}
S_{i k} & =S_{0, k}+S_{m, k} \cos \omega\left(t_{i}-t_{0}\right)+Z_{m, k} \sin \omega\left(t_{i}-t_{0}\right) \\
& =S_{0, k}+Y_{m, k} \cos \omega\left(t_{i}-t^{*}\right) .
\end{aligned}
$$

For signals induced by DM particles one would expect: (i) $Z_{m, k} \sim 0$ (because of the orthogonality between the cosine and the sine functions); (ii) $S_{m, k} \simeq Y_{m, k}$; (iii) $t^{*} \simeq t_{0}=$ 152.5 day. In fact, these conditions hold for most of the dark halo models; however, as mentioned above, slight differences can be expected in case of possible contributions from non-thermalized DM components, such as e.g. the SagDEG stream [15] and the caustics [44].

Figure 7-left shows the $2 \sigma$ contours in the plane $\left(S_{m}, Z_{m}\right)$ for the (2-6) $\mathrm{keV}$ and (6-14) $\mathrm{keV}$ energy intervals and

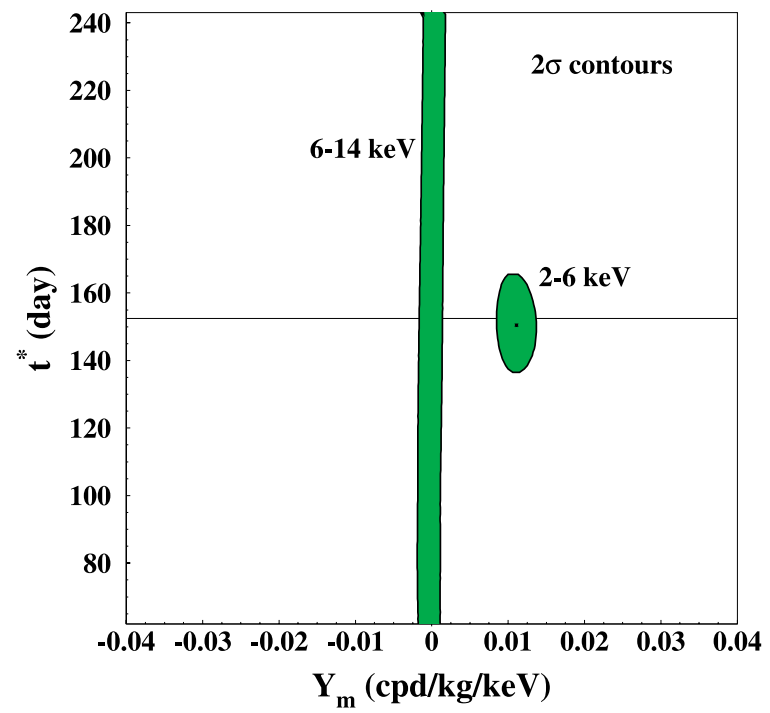

considering the cumulative exposure of 1.17 ton $\times$ yr. A modulation amplitude is present in the lower energy intervals and the phase agrees with that expected for DM induced signals 
Table 5 Best fit values for the (2-6) and (6-14) keV energy interval ( $\sigma \sigma$ errors) for $\mathrm{S}_{m}$ versus $\mathrm{Z}_{m}$ and $Y_{m}$ versus $t^{*}$, considering the cumulative exposure of 1.17 ton $\times$ yr. See also Fig. 7

\begin{tabular}{lllll}
\hline$E$ & $S_{m}$ & $Z_{m}$ & $Y_{m}$ & $t^{*}$ \\
$(\mathrm{keV})$ & $(\mathrm{cpd} / \mathrm{kg} / \mathrm{keV})$ & $(\mathrm{cpd} / \mathrm{kg} / \mathrm{keV})$ & $(0.0111 \pm 0.0013)$ & $(150.5 \pm 7.0)$ \\
\hline $2-6$ & $(0.0111 \pm 0.0013)$ & $-(0.0004 \pm 0.0014)$ & $-(0.0001 \pm 0.0008)$ & Undefined \\
$6-14$ & $-(0.0001 \pm 0.0008)$ & $(0.0002 \pm 0.0005)$ & \\
\hline
\end{tabular}

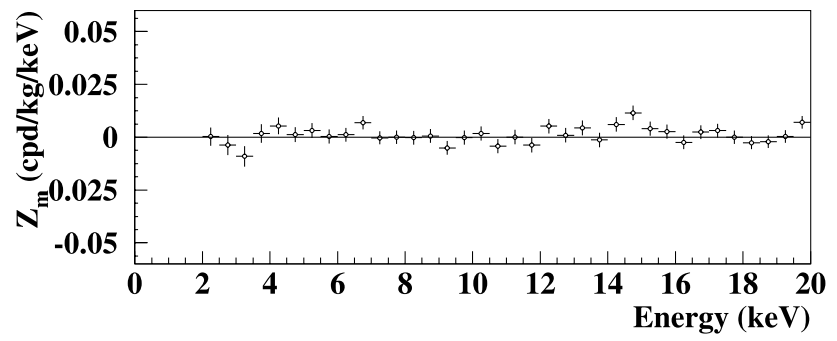

Fig. 8 Energy distribution of the $Z_{m}$ variable for the total exposure (1.17 ton $\times$ yr, DAMA/NaI\&DAMA/LIBRA), once forced to zero the contribution of the cosine function in (1). The energy bin is 0.5 $\mathrm{keV}$. The $Z_{m}$ values are expected to be zero in case of presence of a DM particles' signal with $t^{*} \simeq t_{0}=152.5$ day. By the fact, the $\chi^{2}$ test applied to the data supports the hypothesis that the $Z_{m}$ values are simply fluctuating around zero; see text

Fig. 7-right shows, instead, those in the plane $\left(Y_{m}, t^{*}\right)$. Table 5 shows the best fit values for the (2-6) and (6-14) $\mathrm{keV}$ energy interval ( $1 \sigma$ errors) for $\mathrm{S}_{m}$ versus $\mathrm{Z}_{m}$ and $Y_{m}$ versus $t^{*}$.

Finally, forcing to zero the contribution of the cosine function in (1), the $Z_{m}$ values as function of the energy have also been determined by using the same procedure. The values of $Z_{m}$ as a function of the energy is reported in Fig. 8. Obviously, such values are expected to be zero in case of presence of a DM signal with $t^{*} \simeq t_{0}=152.5$ day. By the fact, the $\chi^{2}$ test applied to the data supports the hypothesis that the $Z_{m}$ values are simply fluctuating around zero; in fact, for example in the (2-14) $\mathrm{keV}$ and (2-20) $\mathrm{keV}$ energy region the $\chi^{2} /$ d.o.f. are equal to $21.6 / 24$ and $47.1 / 36$ (probability of $60 \%$ and $10 \%$ ), respectively.

The behaviors of the $Y_{m}$ and of the phase $t^{*}$ variables as function of energy are shown in Fig. 9 for the total exposure (1.17 ton $\times$ yr, DAMA/NaI\&DAMA/LIBRA). The $Y_{m}$ are superimposed with the $S_{m}$ values with $1 \mathrm{keV}$ energy bin (unlike Fig. 6 where the energy bin is $0.5 \mathrm{keV}$ ). As in the previous analyses, an annual modulation effect is present in the lower energy intervals and the phase agrees with that expected for DM induced signals.

These results confirm those achieved by other kinds of analyses.

Sometimes naive statements were put forwards as the fact that in nature several phenomena may show some kind of periodicity. It is worth noting that the point is
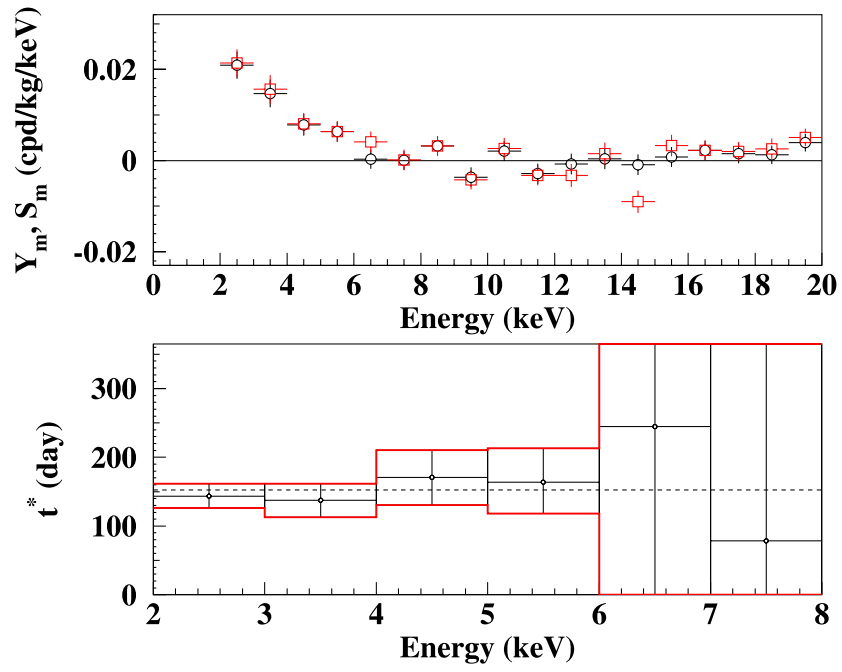

Fig. 9 (Color online) Top: Energy distributions of the $Y_{m}$ variable (light data points; red) and of the $S_{m}$ variable (solid data points) for the total exposure (1.17 ton $\times$ yr, DAMA/NaI\&DAMA/LIBRA). Here, unlike the data of Fig. 6, the energy bin is $1 \mathrm{keV}$. Bottom: Energy distribution of the phase $t^{*}$ for the total exposure; here the errors are at $2 \sigma$. An annual modulation effect is present in the lower energy intervals up to $6 \mathrm{keV}$ and the phase agrees with that expected for DM induced signals. No modulation is present above $6 \mathrm{keV}$ and the phase is undetermined

whether they might mimic the annual modulation signature in DAMA/LIBRA (and former DAMA/NaI), i.e. whether they might be not only quantitatively able to account for the observed modulation amplitude but also able to contemporaneously satisfy all the requirements of the DM annual modulation signature. The same is also for side reactions. This has already been deeply investigated in Refs. [31, 32] and references therein; the arguments and the quantitative conclusions, presented there, also apply to the DAMA/LIBRA5,6 data. Some additional arguments have also been recently addressed in $[43,45,46]$.

\section{Comments}

The obtained model independent evidence-at $8.9 \sigma$ C.L. over 13 annual cycles-is compatible with a wide set of scenarios regarding the nature of the DM candidate and related astrophysical, nuclear and particle Physics (see e.g. 
Refs. [12-14, 16-19], Appendix A of Ref. [31] and in literature, for example see [36-38, 47-52]); and many other possibilities are open. Further future works are foreseen.

It is worth recalling that no other experiment exists, whose result can be directly compared in a model-independent way with those by DAMA/NaI and DAMA/LIBRA, and that-more in general-results obtained with different target materials and/or different approaches cannot be directly compared among them in a model-independent way. This is in particular due to the existing experimental and theoretical uncertainties, not last e.g. how many kinds of dark matter particles can exist in the Universe,$^{2}$ the nature, the interaction types, the different nuclear and/or atomic correlated aspects, the unknown right halo model, the right DM density, etc. as well as the uncertainties on the values of each one of the many involved experimental and theoretical parameter/assumption/approximation used in the calculations. Moreover, some experimental aspects of some techniques used in the field have also to be addressed [12, 45, 46, 53]. Another relevant argument is the methodological robustness [54]. In particular, the general considerations on comparisons reported in Appendix A of Ref. [31] still hold. Hence, claims for contradiction have no scientific basis. On the other hand, whatever possible "positive" result has to be interpreted and a large room of compatibility with DAMA annual modulation evidence is present.

Similar considerations can also be done for the indirect detection searches, since it does not exist a biunivocal correspondence between the observables in the direct and indirect experiments. However, if possible excesses in the positron to electron flux ratio and in the $\gamma$ rays flux with respect to a modeling of the background contribution, which is expected from the considered sources, might be interpreted-under some assumptions-in terms of Dark Matter, this would also be not in conflict with the effect observed by DAMA experiments. It is worth noting that different possibilities either considering different background modeling or accounting for other kinds of sources can also explain the indirect observations [55-59].

Finally, as regards the accelerator searches for new particles beyond the Standard Model of particle Physics, it is worth noting that they can demonstrate the existence of some of the possible DM candidates, but cannot credit that a certain particle is the DM solution or the "single" DM solution. Moreover, DM candidates and scenarios exist (even e.g. for the neutralino candidate) on which accelerators cannot give any information. It is also worth noting that for

\footnotetext{
${ }^{2}$ In fact, it is worth noting that, considering the richness in particles of the visible matter which is less than $1 \%$ of the Universe density, one could also expect that the particle part of the Dark Matter in the Universe may also be multicomponent.
}

every candidate (including the neutralino) there exist various different possibilities for the theoretical aspects. Nevertheless, the results from accelerators will give outstanding and crucial complementary information in the field.

A new upgrade of DAMA/LIBRA is foreseen in 2010 with the replacement of all the low background PMTs with new ones having higher quantum efficiency; the main aim is to lower the software energy threshold and, thus, to increase the experimental sensitivity and to disentangle-in the corollary investigation on the candidate particle(s) - at least some of the many possible astrophysical, nuclear and particle Physics scenarios and related experimental and theoretical uncertainties.

\section{Conclusions}

The new annual cycles DAMA/LIBRA-5,6 have further confirmed a peculiar annual modulation of the single-hit events in the (2-6) $\mathrm{keV}$ energy region satisfying the many requests of the DM annual modulation signature; the total exposure by former DAMA/NaI and present DAMA/LIBRA is 1.17 ton $\times$ yr.

In fact, as required by the DM annual modulation signature: (1) the single-hit events show a clear cosine-like modulation as expected for the DM signal; (2) the measured period is equal to $(0.999 \pm 0.002)$ yr well compatible with the $1 \mathrm{yr}$ period as expected for the DM signal; (3) the measured phase $(146 \pm 7)$ days is well compatible with the roughly $\simeq 152.5$ days expected for the DM signal; (4) the modulation is present only in the low energy (2-6) keV interval and not in other higher energy regions, consistently with expectation for the DM signal; (5) the modulation is present only in the single-hit events, while it is absent in the multiple-hit ones as expected for the DM signal; (6) the measured modulation amplitude in $\mathrm{NaI}(\mathrm{Tl})$ of the single-hit events in the (2-6) $\mathrm{keV}$ energy interval is: $(0.0116 \pm 0.0013) \mathrm{cpd} / \mathrm{kg} / \mathrm{keV}(8.9$ $\sigma$ C.L.). No systematic or side processes able to simultaneously satisfy all the many peculiarities of the signature and to account for the whole measured modulation amplitude is available. Further work is in progress.

\section{References}

1. P. Belli, R. Bernabei, C. Bacci, A. Incicchitti, R. Marcovaldi, D. Prosperi, DAMA proposal to INFN Scientific Committee II, April 24th 1990

2. R. Bernabei et al., Phys. Lett. B 389, 757 (1996)

3. R. Bernabei et al., Phys. Lett. B 424, 195 (1998)

4. R. Bernabei et al., Phys. Lett. B 450, 448 (1999)

5. P. Belli et al., Phys. Rev. D 61, 023512 (2000)

6. R. Bernabei et al., Phys. Lett. B 480, 23 (2000)

7. R. Bernabei et al., Phys. Lett. B 509, 197 (2001)

8. R. Bernabei et al., Eur. Phys. J. C 23, 61 (2002) 
9. P. Belli et al., Phys. Rev. D 66, 043503 (2002)

10. R. Bernabei et al., Il Nuovo Cimento A 112, 545 (1999)

11. R. Bernabei et al., Eur. Phys. J. C 18, 283 (2000)

12. R. Bernabei et al., Riv. Nuovo Cimento 26(1), 1-73 (2003)

13. R. Bernabei et al., Int. J. Mod. Phys. D 13, 2127 (2004)

14. R. Bernabei et al., Int. J. Mod. Phys. A 21, 1445 (2006)

15. R. Bernabei et al., Eur. Phys. J. C 47, 263 (2006)

16. R. Bernabei et al., Int. J. Mod. Phys. A 22, 3155 (2007)

17. R. Bernabei et al., Eur. Phys. J. C 53, 205 (2008)

18. R. Bernabei et al., Phys. Rev. D 77, 023506 (2008)

19. R. Bernabei et al., Mod. Phys. Lett. A 23, 2125 (2008)

20. R. Bernabei et al., Phys. Lett. B 408, 439 (1997)

21. P. Belli et al., Phys. Lett. B 460, 236 (1999)

22. R. Bernabei et al., Phys. Rev. Lett. 83, 4918 (1999)

23. P. Belli et al., Phys. Rev. C 60, 065501 (1999)

24. R. Bernabei et al., Il Nuovo Cimento A 112, 1541 (1999)

25. R. Bernabei et al., Phys. Lett. B 515, 6 (2001)

26. F. Cappella et al., Eur. Phys. J. C 14, 1 (2002)

27. R. Bernabei et al., Eur. Phys. J. A 23, 7 (2005)

28. R. Bernabei et al., Eur. Phys. J. A 24, 51 (2005)

29. R. Bernabei et al., Astropart. Phys. 4, 45 (1995)

30. R. Bernabei, The Identification of Dark Matter (World Scientific, Singapore, 1997), p. 574

31. R. Bernabei et al., Eur. Phys. J. C 56, 333 (2008)

32. R. Bernabei et al., Nucl. Instrum. Methhods A 592, 297 (2008)

33. R. Bernabei et al., Eur. Phys. J. C 62, 327 (2009)

34. K.A. Drukier et al., Phys. Rev. D 33, 3495 (1986)

35. K. Freese et al., Phys. Rev. D 37, 3388 (1988)

36. D. Smith, N. Weiner, Phys. Rev. D 64, 043502 (2001)

37. D. Tucker-Smith, N. Weiner, Phys. Rev. D 72, 063509 (2005)

38. D.P. Finkbeiner et al., Phys. Rev. D 80, 115008 (2009)
39. K. Freese et al., Phys. Rev. D 71, 043516 (2005)

40. K. Freese et al., Phys. Rev. Lett. 92, 111301 (2004)

41. W.H. Press, G.B. Rybicki, Astrophys. J. 338, 277 (1989)

42. J.D. Scargle, Astrophys. J. 263, 835 (1982)

43. R. Bernabei et al., in Proc. Int. Conf. SciNeGHE 2009, Assisi, Italy, October 2009, arXiv:0912.0660 [astro-ph.GA] (2009, to appear)

44. F.S. Ling, P. Sikivie, S. Wick, Phys. Rev. D 70, 123503 (2004)

45. R. Bernabei et al., J. Phys., Conf. Ser. 203, 012040 (2010); arXiv:0912.4200

46. R. Bernabei et al., http://taup2009.lngs.infn.it/slides/jul3/ nozzoli.pdf, talk given by F. Nozzoli

47. A. Bottino, N. Fornengo, S. Scopel, Phys. Rev. D 67, 063519 (2003)

48. A. Bottino, F. Donato, N. Fornengo, S. Scopel, Phys. Rev. D 69, $037302(2003)$

49. A. Bottino, F. Donato, N. Fornengo, S. Scopel, Phys. Rev. D 78, 083520 (2008)

50. A. Bottino, F. Donato, N. Fornengo, S. Scopel, arXiv:0912.4025

51. R. Foot, Phys. Rev. D 78, 043529 (2008)

52. Y. Bai, P.J. Fox, J. High Energy Phys. 0911, 052 (2009)

53. R. Bernabei et al., in Liquid Noble Gases for Dark Matter Searches: a Synoptic Survey (Exorma Ed., Rome, 2009) pp. 1-53, arXiv:0806.0011v2, ISBN 978-88-95688-12-1

54. R. Hudson, Found. Phys. 39, 174 (2009)

55. F. Donato et al., Phys. Rev. Lett. 102, 071301 (2009)

56. T. Delahaye et al., Astron. Astrophys. 501, 821 (2009)

57. S. Profumo, arXiv:0812.4457

58. P. Blasi, Phys. Rev. Lett. 103, 051104 (2009)

59. M. Ahlers et al., Phys. Rev. D 80, 123017 (2009) 